\title{
Efficacy and Safety of Alitretinoin Therapy in Korean Elderly Patients with Chronic Hand Eczema: A Retrospective Single Center Study
}

\author{
Mingyul Jo, Ho Song Kang ${ }^{1}$, Min-Soo Kim, Hyang-Joon Park², Mihn-Sook Jue \\ Department of Dermatology, Veterans Health Service Medical Center, ${ }^{1}$ Division of Dermatology, Department of Medicine, \\ Hanyang University Graduate School, Seoul, ${ }^{2}$ Department of Dermatology, Gachon University of Medicine and Science, Incheon, Korea
}

Background: Chronic hand eczema (CHE) tends to be refractory to conventional therapy. Previous clinical trials have found that alitretinoin is an effective and well-tolerated treatment for $\mathrm{CHE}$. However, there is a relative lack of data on the effectiveness of alitretinoin in elderly patients. Objective: The aim of this study was to investigate the efficacy and safety of oral alitretinoin in elderly patients with moderate to severe $\mathrm{CHE}$ in Korea. Methods: We retrospectively investigated 46 CHE patients who were treated with either $10 \mathrm{mg}$ or $30 \mathrm{mg}$ of alitretinoin between June of 2016 and July of 2018. The physician's global assessment (PGA) was used to evaluate treatment efficacy. All adverse events were retrospectively evaluated with respect to laboratory testing, including complete blood cell count, fasting blood chemistry, lipid profile, and liver and thyroid function tests. Results: The mean patient age in this study was $71.0 \pm 5.1$ years. The treatment period was over eight weeks. A total of 38 of 46 patients (82.6\%) exhibited clinical improvement with PGA ratings of 'clear' or 'almost clear.' There were 13 patients (28.3\%) who experienced an adverse effect, with the most common being headache $(13.0 \%)$ and gastrointestinal symptoms $(8.7 \%)$ followed by xerosis $(6.5 \%)$. A total of 13 patients developed or

Received May 16, 2019, Revised June 24, 2019, Accepted for publication July 31, 2019

Corresponding author: Mihn-Sook Jue, Veterans Health Service Medical Center, 53 Jinhwangdo-ro 61-gil, Gangdong-gu, Seoul 05368, Korea. Tel: 82-2-2225-1388, Fax: 82-2-2225-4374, E-mail: zooooz@hanmail.net ORCID: https://orcid.org/0000-0002-8253-6188

This is an Open Access article distributed under the terms of the Creative Commons Attribution Non-Commercial License (http://creativecommons. org/licenses/by-nc/4.0) which permits unrestricted non-commercial use, distribution, and reproduction in any medium, provided the original work is properly cited.

Copyright (c) The Korean Dermatological Association and The Korean Society for Investigative Dermatology exhibited worsening hypertriglyceridemia (28.3\%). Conclusion: Alitretinoin can be considered a safe and effective treatment option in elderly patients with moderate to severe CHE. (Ann Dermatol 31(6) $595 \sim 600,2019$ )

\section{-Keywords-}

Alitretinoin, Efficacy, Elderly, Hand eczema, Korea, Safety

\section{INTRODUCTION}

Chronic hand eczema (CHE) is an inflammation of hand skin that persists for more than three months. CHE is a common inflammatory skin condition that has a significant and negative impact on the affected individual and society ${ }^{1}$. The lifetime prevalence of $\mathrm{CHE}$ in the general population is approximately $15 \%$, with $5 \%$ to $7 \%$ developing severe $\mathrm{CHE}^{2,3}$. Mild cases of $\mathrm{CHE}$ are typically managed with emollients and topical steroids ${ }^{4}$. On the other hand, severe $\mathrm{CHE}$ can be debilitating because it is often refractory to emollients and topical steroids. There are a few treatment options available for patients who do not respond adequately to topical corticosteroids; however, there is limited evidence from clinical trials on their efficacy. Alitretinoin (9-cis retinoic acid) is a newly developed retinoid with anti-inflammatory properties that received its first national regulatory approval in the UK and recently licensed by the USA Food and Drug Administration as a systemic agent for severe $\mathrm{CHE}$ refractory to topical corticosteroid therapy ${ }^{5,6}$. Although there have been many studies on the epidemiology of $\mathrm{CHE}$, most of these studies have focused on patients between the ages of 10 and 60 years, with limited data on elderly patients. With increasing adult lifespans, the proportion of the elderly population can be 
expected to increase, along with a concomitant increase in the incidence of $\mathrm{CHE}$ in the elderly. In addition, due to age-associated changes in the skin barrier, the risk of contact dermatitis is likely to increase ${ }^{7}$. Therefore, there is an emerging need to study $\mathrm{CHE}$ in the elderly population. Although alitretinoin has been approved for the treatment of $\mathrm{CHE}$, and several studies have demonstrated its effectiveness in $\mathrm{CHE}^{4,8-12}$, there is limited data regarding its safety and efficacy in elderly patients. In the present study, we considered that the elderly, a group predisposed to fragile skin and easy bruising, may experience unacceptable side effects from long-term maintenance with potent topical steroids. Alitretinoin may be particularly helpful in this patient population. Thus, the purpose of this study was to investigate the efficacy and safety of oral alitretinoin in elderly patients with $\mathrm{CHE}$ in Korea.

\section{MATERIALS AND METHODS}

\section{Study design}

This retrospective study was conducted in the Department of Dermatology at the Veterans Health Service Medical Center and was approved and monitored by the Institutional Review Board (IRB) of the Veterans Health Service Medical Center, Korea (IRB no. 2018-02-016). CHE was defined as hand eczema that had persisted for at least three months. Patients who were diagnosed with moderate to severe $\mathrm{CHE}$ refractory to topical steroid treatment were included in this study. We reviewed the medical records of $46 \mathrm{CHE}$ patients over 60 years old who were treated with alitretinoin between June 2016 and July 2018. Baseline severity was classified into five categories (clear, almost clear, mild, moderate, and severe) according to the Physician's global assessment (PGA) ${ }^{4}$. Laboratory monitoring was performed for all patients while they were being treated with alitretinoin. Blood lipids, thyroid function, and liver function parameters were used to identify laboratory abnormalities. Eighteen patients with mild hypertriglyceridemia were included in this study and they were carefully monitored for dyslipidemia. One patient who had undergone a thyroidectomy due to thyroid cancer was included in this study. She was treated with alitretinoin during continuous follow-up with regular endocrinology consultation. The recommended start and maintenance dose of alitretinoin was $30 \mathrm{mg} / \mathrm{d}$. If adverse events occurred, a dose reduction to $10 \mathrm{mg} / \mathrm{d}$ was allowed. Patients were also permitted to use medium potency topical corticosteroids and emollients while being treated with alitretinoin.

\section{Clinical assessment of efficacy and safety}

A medical record review was performed retrospectively utilizing clinical photography. We also investigated treatment characteristics, including prescription period, treatment effectiveness, side effects, and laboratory abnormalities. Treatment efficacy was evaluated retrospectively eight weeks after initiation of treatment according to PGA score, the primary efficacy parameter. A response was defined as a PGA assessment of either "clear" or "almost clear". Laboratory tests (complete blood cell count, fasting blood chemistry, lipid profile and thyroid function test) were performed before initiation of treatment and after 8 weeks of treatment. Triglyceride concentrations were categorized as lower than $150 \mathrm{mg} / \mathrm{dl}$ (normal), 150 to lower than 499 $\mathrm{mg} / \mathrm{dl}$ (mild hypertriglyceridemia), 500 to lower than 886 $\mathrm{mg} / \mathrm{dl}$ (moderate hypertriglyceridemia), $886 \mathrm{mg} / \mathrm{dl}$ or higher (severe hypertriglyceridemia). Normal low density lipoprotein cholesterol (LDL-C) concentrations were defined as lower than $100 \mathrm{mg} / \mathrm{dl}^{13}$.

\section{Statistical analysis}

Data from all available patients were analyzed descriptively. Response rates according to baseline severity and types of hand eczema were analyzed using Fisher's exact test. The statistics program IBM SPSS Statistics ver. 18.0 (IBM Corp., Armonk, NY, USA) was used to analyze data. A $p<0.05$ was considered statistically significant.

\section{RESULTS}

\section{Patients}

A total of 46 patients aged 60 or older with CHE were included in this study. Among the 46 patients involved in the study, 41 were male and 5 were female. The mean age was $71.0 \pm 5.1$ years. Patient demographics and characteristics are shown in Table 1. Patients were classified into hyperkeratotic, vesicular (pompholyx), or fingertip hand eczema groups according to the German guidelines $^{14}$. In total, $71.7 \%$ (33/46) of patients presented with hyperkeratotic type hand eczema. Ten patients $(21.7 \%)$ exhibited vesicular type hand eczema and four patients (8.7\%) exhibited fingertip type CHE. One patient had both hyperkeratotic and vesicular type hand eczema. According to the baseline PGA score, 33 patients (71.7\%) were classified as moderate and 13 patients (28.3\%) were classified as severe.

\section{Efficacy}

The average treatment period was $180.4 \pm 114.6$ days. Among a total of 46 patients, $38(82.6 \%)$ responded to 
Table 1. Patient demographics

\begin{tabular}{|c|c|}
\hline Characteristic & Value \\
\hline Mean age $(y r)$ & $71.0 \pm 5.1$ \\
\hline \multicolumn{2}{|l|}{ Sex } \\
\hline Male & $41(89.1)$ \\
\hline Female & $5(10.9)$ \\
\hline \multicolumn{2}{|l|}{ Past medical history* } \\
\hline Hypertension & $30(65.2)$ \\
\hline Diabetes mellitus & $19(41.3)$ \\
\hline Chronic kidney disease & $3(6.5)$ \\
\hline Dyslipidemia & $18(39.1)$ \\
\hline Fatty liver & $5(10.9)$ \\
\hline Hypothyroidism & $1(2.2)$ \\
\hline Average disease duration (mo) & $48.5 \pm 49.5$ \\
\hline Average prescription period (d) & $180.4 \pm 114.6$ \\
\hline \multicolumn{2}{|l|}{ Clinical subtypes* } \\
\hline Hyperkeratotic & $33(71.7)$ \\
\hline Vesicular & $10(21.7)$ \\
\hline Fingertip & $4(8.7)$ \\
\hline \multicolumn{2}{|l|}{ PGA at baseline } \\
\hline Clear & 0 \\
\hline Almost clear & 0 \\
\hline Mild & 0 \\
\hline Moderate & $33(71.7)$ \\
\hline Severe & $13(28.3)$ \\
\hline \multicolumn{2}{|l|}{ Reason for discontinuation } \\
\hline Ineffectiveness & $3(6.5)$ \\
\hline Adverse events & $5(10.9)$ \\
\hline Cleared hand eczema & $8(17.4)$ \\
\hline Others & $6(13.0)$ \\
\hline
\end{tabular}

Values are presented as mean \pm standard deviation or number (\%). PGA: physician's global assessment. *Multiple nominations possible.

Table 2. Summary of PGA after alitretinoin treatment

\begin{tabular}{lc}
\hline Summary of PGA & Number (\%) \\
\hline Total patients & $46(100)$ \\
Outcome & \\
Clear & $21(45.6)^{*}$ \\
Almost clear & $17(37.0)^{*}$ \\
Mild & $3(6.5)$ \\
Moderate & $5(10.9)$ \\
\hline
\end{tabular}

PGA: physician's global assessment. *Responseresponders, defined as 'clear' or 'almost clear' hands.

treatment, with PGA ratings of 'clear' or 'almost clear' (Table 2). According to baseline severity (Fig. 1), patients with moderate CHE $(84.8 \%, 28 / 33)$ exhibited better clinical improvement than those with severe CHE $(76.9 \%$, $10 / 13)$, although the difference was not statistically significant $(p=0.6689)$. With respect to CHE type (Table 3$)$, the response rate was highest in the fingertip type $(100 \%$, $4 / 4)$, followed by hyperkeratotic $(81.8 \%, 27 / 33)$ and vesic-

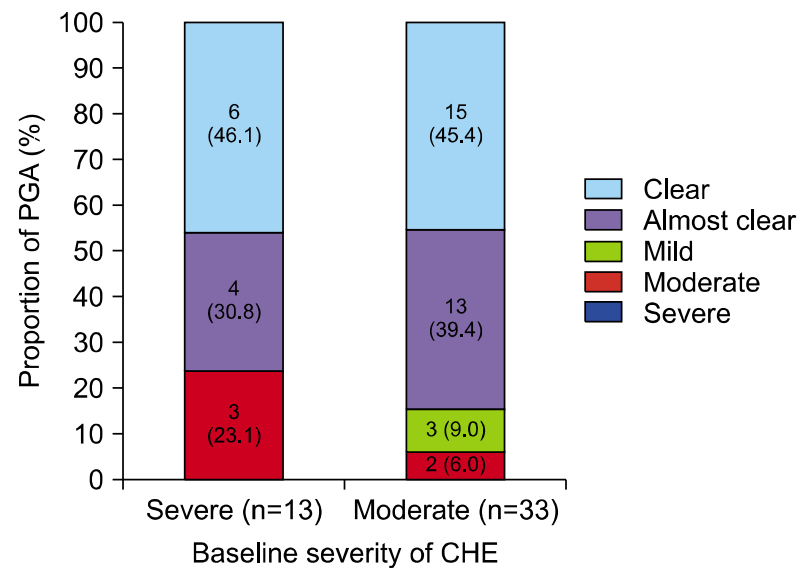

Fig. 1. Evaluation of physician's global assessment (PGA) according to severity of chronic hand eczema $(\mathrm{CHE})$ at baseline.

Table 3. Responding patients according to type of chronic hand eczema after alitretinoin treatment

\begin{tabular}{lc}
\hline Classification & Number $(\%)$ \\
\hline Hyperkeratotic & $33(100)$ \\
Total & $27(81.8)$ \\
Responders* & \\
Vesicular & $10(100)$ \\
Total & $8(80.0)$ \\
Responders* & \\
Finger tip & $4(100)$ \\
Total & $4(100)$ \\
Responders* &
\end{tabular}

*Defined as 'clear' or 'almost clear' hands.

ular types $(80.0 \%, 8 / 10)$. However, the response rate to alitretinoin according to CHE type was not statistically significant $(p=1)$. Eight patients $(17.4 \%)$ discontinued alitretinoin treatment because of cleared HE (clear/almost clear). Three patients $(6.5 \%)$ stopped treatment because of ineffectiveness.

\section{Safety}

A total of 13 patients experienced an adverse event $(28.3 \%, 13 / 46)$. Adverse events resulted in discontinuation of therapy in five patients $(10.9 \%, 5 / 46)$, which consisted of headache $(4.3 \%, 2 / 46)$, xerosis $(4.3 \%, 2 / 46)$, and gastrointestinal (Gl) symptoms $(2.2 \%, 1 / 46)$ (Table 4$)$. Abnormalities in laboratory tests were limited to typical retinoid effects and were observed in 13 patients (Table 5), all of which exhibited an elevated triglyceride level $(28.3 \%$, 13/46), with three of them also exhibiting an elevated LDL-C level range from 46 to $51 \mathrm{mg} / \mathrm{dl}$ above their premeasured baseline. In eight patients with a normal triglyceride level at baseline, mild hypertriglyceridemia was ob- 
served after alitretinoin treatment. Moderate hypertriglyceridemia was observed in one patient who had a normal triglyceride level at baseline. Four patients with mild hypertriglyceridemia at baseline exhibited an elevated triglyceride level from 240 up to $340 \mathrm{mg} / \mathrm{dl}$ above their premeasured baseline. There were no instances of liver enzyme elevation or thyroid dysfunction.

\section{DISCUSSION}

The efficacy and side effects of alitretinoin have been studied in several large, well-designed trials (Table 6) ${ }^{4,9,11}$. According to a meta-analysis based on these studies, alitretinoin was well tolerated and showed good efficacy in treatment of $\mathrm{CHE}^{15}$. Although many of these studies have demonstrated a high therapeutic efficacy of alitretinoin in $\mathrm{CHE}$, data on the efficacy and safety of alitretinoin in the elderly is lacking. Indeed, the mean ages of patients in

Table 4. Adverse events during treatment with alitretinoin

\begin{tabular}{llc}
\hline Adverse event* & Total & Discontinued \\
\hline Headache & $6(13.0)$ & 2 \\
Nausea/vomiting & $4(8.7)$ & 1 \\
Dryness & $3(6.5)$ & 2 \\
Dizziness & $1(2.1)$ & 0 \\
\hline
\end{tabular}

Values are presented as number (\%) or number only. *Multiple nominations possible. previous studies on alitretinoin have typically been in the 40 s to 50 s, while the average age in this study was 71 years $^{4,6,11,12,15}$.

In our study of 46 patients, $38(82.6 \%)$ responded to treatment with alitretinoin, which was almost twice the response rate of previous studies ${ }^{4,5,9,11}$. We explain the superior effectiveness of alitretinoin observed in our study as follows. First, compared with previous studies, the mean age of the patients included in this study was 20 to 30 years older, and most patients were male. As these were the most significant differences between our study and previous studies, they may be at least partially associated with the difference in efficacy. For example, polypharmacy and physiological changes to liver and kidney function, which are commonly seen in elderly patients, might affect alitretinoin metabolism, thereby increasing its efficacy. Although alitretinoin does not exhibit increased efficacy with weight-adjusted dosing, it is possible that decreased lean body mass and increased body fat mass associated

Table 5. Reported abnormal laboratory findings

\begin{tabular}{lc}
\hline \multicolumn{1}{c}{ Laboratory abnormality* } & Number $(\%)$ \\
\hline Triglyceride elevation & $13(28.3)$ \\
LDL cholesterol elevation & $3(6.5)$ \\
Liver enzyme elevation & $0(0)$ \\
Thyroid dysfunction & $0(0)$ \\
\hline
\end{tabular}

LDL: low-density lipoprotein. ${ }^{*}$ Multiple nominations possible.

Table 6. Comparison of clinical trials of alitretinoin treatment for chronic hand eczema

\begin{tabular}{|c|c|c|c|c|}
\hline Variable & Ruzicka et al. ${ }^{4}$ & Dirschka et al. ${ }^{11}$ & Kwon et al. ${ }^{9}$ & Our study \\
\hline Patients & 409 (100) & 249 (100) & $27(100)$ & $46(100)$ \\
\hline Hyperkeratotic & $349(85.3)$ & $227(91.2)$ & $19(70.3)$ & $33(71.7)$ \\
\hline Vesicular & $111(27.1)$ & $54(21.7)$ & $13(48.1)$ & $10(21.7)$ \\
\hline Fingertip & $196(47.9)$ & $80(32.1)$ & $8(29.6)$ & $4(8.7)$ \\
\hline Others & $55(13.4)$ & $41(16.5)$ & 0 & 0 \\
\hline Average age (yr) & $48 \pm 13$ & $48.1 \pm 13.1$ & $34.8 \pm 11.2$ & $71.0 \pm 5.1$ \\
\hline Male & $223(54.5)$ & $132(53.0)$ & $13(48.1)$ & $41(89.1)$ \\
\hline Responder & $195(47.7)$ & $116(46.6)$ & $12(44.4)$ & $38(82.6)$ \\
\hline \multicolumn{5}{|l|}{ Adverse events } \\
\hline Nasopharyngitis & $24(6)$ & $23(9.3)$ & 0 & 0 \\
\hline Headache & $81(20)$ & $6(2.4)$ & $11(40.7)$ & $6(13.0)$ \\
\hline Dryness & $35(8)$ & - & $3(11.1)$ & $3(6.5)$ \\
\hline Nausea or vomiting & $14(3)$ & $11(4.4)$ & $2(7.4)$ & $4(8.7)$ \\
\hline \multicolumn{5}{|l|}{ Laboratory findings } \\
\hline Cholesterol high & $37(14)$ & $13(9.8)$ & 0 & $3(6.5)$ \\
\hline Triglycerides high & $20(8)$ & $12(9.0)$ & 0 & $13(28.3)$ \\
\hline AST/ALT high & $5(1)$ & $7(2.9)$ & 0 & 0 \\
\hline TSH low & $28(7)$ & $23(10.4)$ & 0 & 0 \\
\hline LDL high & - & $43(32.6)$ & 0 & $3(6.5)$ \\
\hline
\end{tabular}

Values are presented as number (\%) or mean \pm standard deviation. AST: aspartate aminotransferase, ALT: alanine aminotransferase, $\mathrm{TSH}$ : thyroid-stimulating hormone, LDL: low-density lipoprotein, -: not mentioned. 
with aging may have affected its efficacy ${ }^{16}$. Second, alitretinoin is an oral agent, and drug adherence is reported to be higher with oral agents than with topical agents in the elderly because it is easier to access regularly ${ }^{17,18}$. Thus, the higher efficacy observed in elderly $\mathrm{CHE}$ patients may have been due to better medication adherence. This result is especially important in the context of elderly patients who, as opposed to oral medications, exhibit a relatively low adherence to topical treatment compared with younger patients.

The adverse events of alitretinoin observed in our study were consistent with the well-known class effects of oral retinoids and RXR-agonists, including headache, GI symptoms, mucocutaneous events, dizziness, and hyperlipidemia (Table 4, 5). Six patients experienced headaches (6/46, 13.0\%), with two patients discontinuing treatment as a result. However, when these patients resumed treatment with alitretinoin after their headaches had subsided, they no longer complained of the same adverse event. Two others showed improvement of headache without taking analgesics. Compared with the study conducted by Kwon et al. ${ }^{9}$, which included patients in the youngest age group among previous clinical trials, headaches and mucocutaneous symptom occurred less frequently, however, Gl symptoms occurred more in our patients. The patients included in our study were also taking multiple drugs and their medications often included anti-inflammatory drugs, such as NSAIDs. Use of such analgesics by our patients for treatment of unrelated conditions, such as osteoarthritis may have obscured headache incidence while exacerbating Gl symptoms. Therefore, when prescribing alitretinoin in elderly patients, it may be important to emphasize that alitretinoin should be taken with food not only to increase efficacy, but also to reduce GI discomfort.

Laboratory abnormalities were frequently observed in our patients, but were limited to dyslipidemia. Specifically, baseline dyslipidemia was observed in 18 (39.1\%) of the patients included in our study, and was newly developed or worsened in 14 patients $(30.4 \%)$. The incidence of dyslipidemia was considerably higher than that seen in previous studies where the mean age was in the late 40s, suggesting that lipid profiles should be monitored more closely in elderly patients being treated with alitretinoin. In 2015, after analyzing clinical data from several previous studies $^{4,6,10,12}$, Schmith et al. ${ }^{19}$ conducted five hundred clinical trial simulations to evaluate the efficacy and safety of alitretinoin treatment. The results of that study showed that elderly patients experience higher efficacy, faster response, and increased probability of hypertriglyceridemia in response to treatment with alitretinoin, which was consistent with the results of our study. Therefore, clinicians should consider utilizing a shorter treatment duration with alitretinoin in elderly patients to avoid the risk of hypertriglyceridemia.

Prescribing medication for elderly patients has several challenges. Pharmacologically, drug absorption through the GI tract decreases with increasing age. However, while hepatic metabolism and glomerular filtration rates also decrease with increasing age, drug bioavailability increases ${ }^{20}$. In addition, multi-drug regimens and multiple medical problems, which are commonly seen in the geriatric population, expose patients to an increased risk of adverse effects of drug-drug interactions ${ }^{20}$. Thus, the suitability of systemic alitretinoin treatment in elderly patients is a potential concern. The patients included in this study were all aged 60 years or older, and thus our results suggest that it is safe to use alitretinoin in the elderly.

This study had several limitations. The first and most significant limitation was the retrospective study design, which precluded standardization of regular follow-up, laboratory monitoring, and treatment duration. A second limitation of this study was that the assessment of disease severity was made using only the PGA score. However, a previous study demonstrated that there is good agreement between PGA score and modified total lesion symptom score ${ }^{9}$. Therefore, we considered that the assessment of severity through PGA score alone should have adequately reflected the severity of the actual disease. Third, our study consisted of a relatively small sample size compared to previous studies. Nevertheless, this study represents the first clinical trial to evaluate the efficacy and safety of alitretinoin treatment in elderly patients over 60 years of age. Additional studies may be needed to confirm the efficacy and side effects of alitretinoin treatment in elderly patients. In conclusion, the results of our study were consistent with previous clinical trials and demonstrated that oral alitretinoin leads to a clinically significant improvement in disease status in elderly patients with moderate to severe CHE refractory to topical corticosteroids. Therefore, alitretinoin should be considered as an effective and safe option for the treatment of moderate to severe $\mathrm{CHE}$ in the elderly.

\section{CONFLICTS OF INTEREST}

The authors have nothing to disclose.

\section{ORCID}

Mingyul Jo, https://orcid.org/0000-0002-6904-1703

Ho Song Kang, https://orcid.org/0000-0003-4257-2560

Min-Soo Kim, http://orcid.org/0000-0001-6133-6243 
Hyang-Joon Park, http://orcid.org/0000-0002-2143-0080

Mihn-Sook Jue, http://orcid.org/0000-0002-8253-6188

\section{REFERENCES}

1. Van Coevorden AM, Coenraads PJ, Svensson A, Bavinck JN, Diepgen TL, Naldi L, et al.; European Dermato-Epidemiology Network (Eden). Overview of studies of treatments for hand eczema-the EDEN hand eczema survey. $\mathrm{Br} J$ Dermatol 2004;151:446-451.

2. Thyssen JP, Johansen JD, Linneberg A, Menné T. The epidemiology of hand eczema in the general population--prevalence and main findings. Contact Dermatitis 2010;62:7587.

3. Diepgen TL, Agner T, Aberer W, Berth-Jones J, Cambazard $\mathrm{F}$, Elsner $\mathrm{P}$, et al. Management of chronic hand eczema. Contact Dermatitis 2007;57:203-210.

4. Ruzicka T, Lynde CW, Jemec GB, Diepgen T, Berth-Jones J, Coenraads PJ, et al. Efficacy and safety of oral alitretinoin (9-cis retinoic acid) in patients with severe chronic hand eczema refractory to topical corticosteroids: results of a randomized, double-blind, placebo-controlled, multicentre trial. Br J Dermatol 2008;158:808-817.

5. King T, McKenna J, Alexandroff $A B$. Alitretinoin for the treatment of severe chronic hand eczema. Patient Prefer Adherence 2014;8:1629-1634.

6. Ruzicka T, Larsen FG, Galewicz D, Horváth A, Coenraads PJ, Thestrup-Pedersen K, et al. Oral alitretinoin (9-cis-retinoic acid) therapy for chronic hand dermatitis in patients refractory to standard therapy: results of a randomized, doubleblind, placebo-controlled, multicenter trial. Arch Dermatol 2004; 140:1453-1459.

7. Prakash AV, Davis MD. Contact dermatitis in older adults: a review of the literature. Am J Clin Dermatol 2010;11:373381.

8. Politiek K, Christoffers WA, Coenraads PJ, Schuttelaar MA. Alitretinoin and acitretin in severe chronic hand eczema; results from a retrospective daily practice study. Dermatol Ther 2016;29:364-371.

9. Kwon HI, Kim JE, Ko JY, Ro YS. Efficacy and safety of alitretinoin for chronic hand eczema in Korean patients. Ann Dermatol 2016;28:364-370.

10. Fowler JF, Graff O, Hamedani AG. A phase 3, randomized, double-blind, placebo-controlled study evaluating the efficacy and safety of alitretinoin (BAL4079) in the treatment of severe chronic hand eczema refractory to potent topical corticosteroid therapy. J Drugs Dermatol 2014;13:1198-1204.

11. Dirschka T, Reich K, Bissonnette R, Maares J, Brown T, Diepgen TL. An open-label study assessing the safety and efficacy of alitretinoin in patients with severe chronic hand eczema unresponsive to topical corticosteroids. Clin Exp Dermatol 2011;36:149-154.

12. Bissonnette R, Worm M, Gerlach B, Guenther L, Cambazard $F$, Ruzicka $T$, et al. Successful retreatment with alitretinoin in patients with relapsed chronic hand eczema. $\mathrm{Br} J$ Dermatol 2010;162:420-426.

13. Rosenson RS, Kastelein JJP. Hypertriglyceridemia [Internet]. Waltham, MA: UpToDate; 2019 [updated 2019 May 13; cited 2019 July 30]. Available from: https://www.uptodate. com/contents/hypertriglyceridemia.

14. Diepgen TL, Elsner $P$, Schliemann S, Fartasch M, Köllner A, Skudlik C, et al.; Deutsche Dermatologische Gesellschaft. Guideline on the management of hand eczema ICD-10 Code: L20. L23. L24. L25. L30. J Dtsch Dermatol Ges 2009; 7 Suppl 3:S1-S16.

15. Al-Dhubaibi MS, Settin AA. The effectiveness of alitretinoin for the treatment of chronic hand eczema: a meta-analysis. Int J Health Sci (Qassim) 2018;12:70-79.

16. Schmitt-Hoffmann AH, Roos B, Schoetzau A, Leese PT, Meyer I, van de Wetering J, et al. Oral alitretinoin: a review of the clinical pharmacokinetics and pharmacodynamics. Expert Rev Clin Pharmacol 2012;5:373-388.

17. Krejci-Manwaring J, McCarty MA, Camacho F, Carroll CL, Johnson K, Manuel J, et al. Adherence with topical treatment is poor compared with adherence with oral agents: implications for effective clinical use of topical agents. J Am Acad Dermatol 2006;54(5 Suppl):S235-S236.

18. Tanei R. Atopic dermatitis in the elderly. Inflamm Allergy Drug Targets 2009;8:398-404.

19. Schmith GD, Singh R, Gomeni R, Graff O, Hamedani AG, Troughton JS, et al. Use of longitudinal dose-response modeling to support the efficacy and tolerability of alitretinoin in severe refractory chronic hand eczema (CHE). CPT Pharmacometrics Syst Pharmacol 2015;4:255-262.

20. Pesante-Pinto JL. Clinical pharmacology and the risks of polypharmacy in the geriatric patient. Phys Med Rehabil Clin N Am 2017;28:739-746. 